\title{
Split mixed dose
}

National Diabetes Information Clearinghouse (NDIC)

\section{Definitions}

Insulin

Defined by National Diabetes Information Clearinghouse (NDIC)

Injection

Defined by National Diabetes Information Clearinghouse (NDIC)

Source

National Diabetes Information Clearinghouse (U.S.). (2009). The diabetes dictionary. [Bethesda, Md.]: U.S. Dept. of Health and Human Services, National Institutes of Health, National Institute of Diabetes and Digestive and Kidney Diseases, National Diabetes Information Clearinghouse.

Division of a prescribed daily dose of insulin into two or more injections given over the course of the day. 